Bull. Korean Math. Soc. 51 (2014), No. 3, pp. 901-909

http://dx.doi.org/10.4134/BKMS.2014.51.3.901

\title{
AREA OF TRIANGLES ASSOCIATED WITH A CURVE
}

\author{
Dong-Soo Kim And Kyu-Chul Shim
}

\begin{abstract}
It is well known that the area $U$ of the triangle formed by three tangents to a parabola $X$ is half of the area $T$ of the triangle formed by joining their points of contact. In this article, we study some properties of $U$ and $T$ for strictly convex plane curves. As a result, we establish a characterization for parabolas.
\end{abstract}

\section{Introduction}

Let $X=X(s)$ be a unit speed smooth curve in the plane $\mathbb{R}^{2}$ with nonvanishing curvature, and let $A=X(s), A_{i}=X\left(s+h_{i}\right), i=1,2$, be three distinct neighboring points on $X$. Denote by $\ell, \ell_{1}, \ell_{2}$ the tangent lines passing through the points $A, A_{1}, A_{2}$ and by $B, B_{1}, B_{2}$ the intersection points $\ell_{1} \cap \ell_{2}, \ell \cap \ell_{1}$, $\ell \cap \ell_{2}$, respectively. It is well known that the area $U\left(s, h_{1}, h_{2}\right)=\left|\triangle B B_{1} B_{2}\right|$ of the triangle formed by three tangents to a parabola is half of the area $T\left(s, h_{1}, h_{2}\right)=\left|\triangle A A_{1} A_{2}\right|$ of the triangle formed by joining their points of contact $([1])$.

The present article studies whether this property exhaustively characterizes parabolas.

Usually, a regular plane curve $X: I \rightarrow \mathbb{R}^{2}$ defined on an open interval is called convex if, for all $t \in I$, the trace $X(I)$ lies entirely on one side of the closed half-plane determined by the tangent line at $X(t)([2])$.

Hereafter, we will say that a simple convex curve $X$ in the plane $\mathbb{R}^{2}$ is strictly convex if the curve is smooth (that is, of class $C^{(3)}$ ) and is of positive curvature $\kappa$ with respect to the unit normal $N$ pointing to the convex side. Hence, in this case we have $\kappa(s)=\left\langle X^{\prime \prime}(s), N(X(s))\right\rangle>0$, where $X(s)$ is an arclength parametrization of $X$.

For a smooth function $f: I \rightarrow \mathbb{R}$ defined on an open interval, we will also say that $f$ is strictly convex if the graph of $f$ has positive curvature $\kappa$ with respect

Received May 28, 2013; Revised June 26, 2013.

2010 Mathematics Subject Classification. 53A04.

Key words and phrases. triangle, area, parabola, strictly convex curve, plane curvature.

This was supported by Basic Science Research Program through the National Research Foundation of Korea (NRF) funded by the Ministry of Education, Science and Technology (2010-0022926). 
to the upward unit normal $N$. This condition is equivalent to the positivity of $f^{\prime \prime}(x)$ on $I$.

Suppose that $X$ is a strictly convex curve in the plane $\mathbb{R}^{2}$ with the unit normal $N$ pointing to the convex side. For a fixed point $P=A \in X$, and for a sufficiently small $h>0$, consider the line $m$ passing through $P+h N(P)$ which is parallel to the tangent $\ell$ of $X$ at $P$. Let us denote by $A_{1}$ and $A_{2}$ the points where the line $m$ intersects the curve $X$. We denote by $L_{P}(h)$ the length $\left|A_{1} A_{2}\right|$ of the chord $A_{1} A_{2}$.

Let us denote by $\ell_{1}, \ell_{2}$ the tangent lines passing through the points $A_{1}, A_{2}$ and by $B, B_{1}, B_{2}$ the intersection points $\ell_{1} \cap \ell_{2}, \ell \cap \ell_{1}, \ell \cap \ell_{2}$, respectively. We denote by $T_{P}(h), U_{P}(h)$ the area $\left|\triangle A A_{1} A_{2}\right|,\left|\triangle B B_{1} B_{2}\right|$, of triangles, respectively. Then, obviously we have $T_{P}(h)=\frac{h}{2} L_{P}(h)$.

In this paper, first of all, in Section 2 we prove the following:

Theorem 1. Let $X$ denote a strictly convex $C^{(3)}$ curve in the plane $\mathbb{R}^{2}$. Then we have

$$
\lim _{h \rightarrow 0} \frac{T_{P}(h)}{h \sqrt{h}}=\frac{\sqrt{2}}{\sqrt{\kappa(P)}}
$$

and

$$
\lim _{h \rightarrow 0} \frac{U_{P}(h)}{h \sqrt{h}}=\frac{\sqrt{2}}{2 \sqrt{\kappa(P)}} .
$$

Next in Section 3, using Theorem 1 we characterize parabolas as follows.

Theorem 2. Let $X=X(s)$ denote a strictly convex $C^{(3)}$ curve in the plane $\mathbb{R}^{2}$. Suppose that for all $s$ and sufficiently small $h_{i}, i=1,2$, the curve $X$ satisfies

$$
U\left(s, h_{1}, h_{2}\right)=\lambda(s) T\left(s, h_{1}, h_{2}\right) .
$$

Then, we have $\lambda(s)=\frac{1}{2}$ and $X$ is an open part of a parabola.

In [8], Krawczyk showed that for a strictly convex $C^{(4)}$ curve $X=X(s)$ in the plane $\mathbb{R}^{2}$, the following holds:

$$
\lim _{h_{1}, h_{2} \rightarrow 0} \frac{T\left(s, h_{1}, h_{2}\right)}{U\left(s, h_{1}, h_{2}\right)}=2 .
$$

His application of (1.4) states that if a strictly convex $C^{(4)}$ curve $X=X(s)$ in the plane $\mathbb{R}^{2}$ satisfies (1.3), then $\lambda(s)=\frac{1}{2}$ and $X$ is an open part of the graph of a quadratic polynomial.

But, for example, consider a function $f(x)$ given by

$$
y=\frac{2 \sqrt{a} c x+1-\sqrt{4 \sqrt{a} c x+1}}{2 c^{2}},
$$

where $a, c>0$. Then, the function $f$ is defined on $I=\left(-\frac{1}{4 \sqrt{a} c}, \infty\right)$. Its graph $X$ is strictly convex and satisfies (1.3) with $\lambda=\frac{1}{2}$. Note that $X$ is not the 
graph of a quadratic polynomial, but an open part of the parabola given in (3.16) in Section 3.

In [6], the first author and Y. H. Kim established five characterizations of parabolas, which are the converses of well-known properties of parabolas originally due to Archimedes ([10]). In [4] and [5], they also proved the higher dimensional analogues of some results in [6].

For a few characterizations of parabolas or conic sections by some properties of tangent lines, see [3] and [7].

Among the graphs of functions, B. Richmond and T. Richmond established a dozen characterizations of parabolas using elementary techniques ([9]). In [9], parabola means the graph of a quadratic polynomial in one variable.

Finally, we pose a question as follows.

Question 3. Let $X$ be a strictly convex $C^{(3)}$ plane curve. Suppose that for each $P \in X$ there exists a positive number $\epsilon=\epsilon(P)>0$ such that $U_{P}(h)=T_{P}(h) / 2$ for all $h$ with $0<h<\epsilon(P)$. Then, is it an open part of a parabola?

Throughout this article, all curves are of class $C^{(3)}$ and connected, unless otherwise mentioned.

\section{Preliminaries and Theorem 1}

In order to prove Theorem 1, we need the following lemma $([6])$.

Lemma 4. Suppose that $X$ is a strictly convex $C^{(3)}$ curve in the plane $\mathbb{R}^{2}$ with the unit normal $N$ pointing to the convex side. Then we have

$$
\lim _{h \rightarrow 0} \frac{1}{\sqrt{h}} L_{P}(h)=\frac{2 \sqrt{2}}{\sqrt{\kappa(P)}},
$$

where $\kappa(P)$ is the curvature of $X$ at $P$ with respect to the unit normal $N$.

First of all, we give a proof of (1.1) in Theorem 1. Since $T_{P}(h)=\frac{h}{2} L_{P}(h)$, it follows from Lemma 4 that the following holds:

$$
\lim _{h \rightarrow 0} \frac{1}{h \sqrt{h}} T_{P}(h)=\frac{\sqrt{2}}{\sqrt{\kappa(P)}} .
$$

In order to prove (1.2) in Theorem 1, we fix an arbitrary point $P$ on $X$. Then, we may take a coordinate system $(x, y)$ of $\mathbb{R}^{2}: P$ is taken to be the origin $(0,0)$ and $x$-axis is the tangent line $\ell$ of $X$ at $P$. Furthermore, we may regard $X$ to be locally the graph of a non-negative strictly convex function $f: \mathbb{R} \rightarrow \mathbb{R}$ with $f(0)=f^{\prime}(0)=0$. Then $N$ is the upward unit normal.

Since the curve $X$ is of class $C^{(3)}$, the Taylor's formula of $f(x)$ is given by

$$
f(x)=a x^{2}+g(x),
$$

where $2 a=f^{\prime \prime}(0)$ and $g(x)$ is an $O\left(|x|^{3}\right)$ function. From $\kappa(P)=f^{\prime \prime}(0)>0$, we see that $a$ is positive. 
For a sufficiently small $h>0$, we denote by $A_{1}(s, f(s))$ and $A_{2}(t, f(t))$ the points where the line $m: y=h$ meets the curve $X$ with $s<0<t$. Then $f(s)=f(t)=h$ and we get $B_{1}\left(s-h / f^{\prime}(s), 0\right), B_{2}\left(t-h / f^{\prime}(t), 0\right)$ and $B\left(x_{0}, y_{0}\right)$ with

$$
x_{0}=\frac{t f^{\prime}(t)-s f^{\prime}(s)}{f^{\prime}(t)-f^{\prime}(s)}
$$

and

$$
y_{0}=\frac{(t-s) f^{\prime}(t) f^{\prime}(s)+h\left(f^{\prime}(t)-f^{\prime}(s)\right)}{f^{\prime}(t)-f^{\prime}(s)} .
$$

Noting that $L_{P}(h)=t-s$, one obtains

$$
\begin{aligned}
2 U_{P}(h) & =\left\{t-s-\frac{h}{f^{\prime}(t)}+\frac{h}{f^{\prime}(s)}\right\}\left(-y_{0}\right) \\
& =h^{2} \frac{\left(f^{\prime}(t)-f^{\prime}(s)\right)}{-f^{\prime}(s) f^{\prime}(t)}-2 h L_{P}(h)+\frac{-f^{\prime}(s) f^{\prime}(t)}{f^{\prime}(t)-f^{\prime}(s)} L_{P}(h)^{2} .
\end{aligned}
$$

It follows from $(2.5)$ that

$$
2 \frac{U_{P}(h)}{h \sqrt{h}}=\alpha_{P}(h)-2 \frac{L_{P}(h)}{\sqrt{h}}+\frac{1}{\alpha_{P}(h)}\left(\frac{L_{P}(h)}{\sqrt{h}}\right)^{2},
$$

where we denote

$$
\alpha_{P}(h)=\sqrt{h} \frac{\left(f^{\prime}(t)-f^{\prime}(s)\right)}{-f^{\prime}(s) f^{\prime}(t)} .
$$

Finally, we prove a lemma, which together with (2.6) and Lemma 4, completes the proof of (2) in Theorem 1.

Lemma 5. We have the following.

$$
\lim _{h \rightarrow 0} \alpha_{P}(h)=\frac{\sqrt{2}}{\sqrt{\kappa(P)}} .
$$

Proof. Note that

$$
\alpha_{P}(h)=\frac{\beta_{P}(h)}{\gamma_{P}(h)}
$$

where we denote

$$
\beta_{P}(h)=\frac{f^{\prime}(t)-f^{\prime}(s)}{t-s}
$$

and

$$
\gamma_{P}(h)=\frac{-f^{\prime}(s) f^{\prime}(t)}{\sqrt{h}(t-s)} .
$$

Applying mean value theorem to the derivative $f^{\prime}(x)$ of $f(x)$ shows that as $h$ tends to $0, \beta_{P}(h)$ goes to $f^{\prime \prime}(0)=\kappa(P)$. To get the limit of $\gamma_{P}(h)$, we put

$$
\delta_{P}(h)=\frac{f^{\prime}(s) f^{\prime}(t)}{s t}
$$


and

$$
\eta_{P}(h)=\frac{-s t}{\sqrt{h}(t-s)} .
$$

Then, we have

$$
\gamma_{P}(h)=\delta_{P}(h) \eta_{P}(h)
$$

Note that

$$
\lim _{h \rightarrow 0} \delta_{P}(h)=\kappa(P)^{2} .
$$

If we use $L_{P}(h)=t-s, \eta_{P}(h)$ can be written as

$$
\eta_{P}(h)=\left(-\frac{s t}{h}\right) /\left(\frac{L_{P}(h)}{\sqrt{h}}\right)
$$

and the numerator of (2.16) can be decomposed as

$$
-\frac{s t}{h}=\left(\frac{L_{P}(h)}{\sqrt{h}}-\frac{t}{\sqrt{h}}\right) \frac{t}{\sqrt{h}} .
$$

Now, to obtain the limit of $\frac{t}{\sqrt{h}}$, we use (2.2). Recalling that $\kappa(P)=f^{\prime \prime}(0)=$ $2 a$, we have

$$
\frac{t}{\sqrt{h}}=\frac{t}{\sqrt{a t^{2}+g(t)}} .
$$

Since $g(x)$ is an $O\left(|x|^{3}\right)$ function, (2.18) implies that $\lim _{h \rightarrow 0} t / \sqrt{h}=1 / \sqrt{a}$. Hence, together with (2.17), Lemma 4 shows that $\lim _{h \rightarrow 0}(-s t) / h=1 / a$, and hence from (2.16) we get

$$
\lim _{h \rightarrow 0} \eta_{P}(h)=\frac{1}{2 \sqrt{a}} .
$$

Thus, it follows from (2.14) and (2.15) that

$$
\lim _{h \rightarrow 0} \gamma_{P}(h)=2 a \sqrt{a} \text {. }
$$

Using $\kappa(P)=2 a$, together with $(2.9)$ and $(2.10),(2.20)$ completes the proof of Lemma 5 .

\section{Proof of Theorem 2}

In this section, we prove Theorem 2.

Suppose that $X=X(s)$ denote a strictly convex $C^{(3)}$ curve in the plane $\mathbb{R}^{2}$ which satisfies for all $s$ and sufficiently small $h_{i}, i=1,2$,

$$
U\left(s, h_{1}, h_{2}\right)=\lambda(s) T\left(s, h_{1}, h_{2}\right) .
$$

Then, in particular, for all $P=X(s)$ and sufficiently small $h>0$ the curve $X$ satisfies

$$
U_{P}(h)=\lambda(P) T_{P}(h) .
$$

Hence, Theorem 1 implies that $\lambda(P)=\frac{1}{2}$. 
In order to prove the remaining part of Theorem 2, first, we fix an arbitrary point $A$ on $X$. As in Section 1 , we take a coordinate system $(x, y)$ of $\mathbb{R}^{2}$ : $A$ is taken to be the origin $(0,0)$ and $x$-axis is the tangent line $\ell$ of $X$ at $A$. Furthermore, we may regard $X$ to be locally the graph of a non-negative strictly convex function $f: \mathbb{R} \rightarrow \mathbb{R}$ with $f(0)=f^{\prime}(0)=0$ and $2 a=f^{\prime \prime}(0)>0$.

For sufficiently small $|s|$ and $|t|$ with $0<s<t$ or $t<s<0$, we let $A_{1}=(s, f(s)), A_{2}=(t, f(t))$ be two neighboring points of $A$ on $X$. Then, the area $T(s, t)$ of the triangle $\triangle A A_{1} A_{2}$ is given by

$$
2 \epsilon T(s, t)=(s f(t)-t f(s)),
$$

where $\epsilon=1$ if $0<s<t$ and $\epsilon=-1$ if $t<s<0$.

Denote by $\ell, \ell_{1}, \ell_{2}$ the tangent lines passing through the points $A, A_{1}, A_{2}$ and by $B, B_{1}, B_{2}$ the intersection points $\ell_{1} \cap \ell_{2}, \ell \cap \ell_{1}, \ell \cap \ell_{2}$, respectively. Then we have $B_{1}\left(s-f(s) / f^{\prime}(s), 0\right), B_{2}\left(t-f(t) / f^{\prime}(t), 0\right)$ and $B\left(x_{0}, y_{0}\right)$ with

$$
y_{0}=\frac{(t-s) f^{\prime}(t) f^{\prime}(s)+f(s) f^{\prime}(t)-f^{\prime}(s) f(t)}{f^{\prime}(t)-f^{\prime}(s)} .
$$

Hence the area $U(s, t)$ of the triangle $\triangle B B_{1} B_{2}$ is given by

$$
\begin{aligned}
2 \epsilon U(s, t) & =\left\{t-s-\frac{f(t)}{f^{\prime}(t)}+\frac{f(s)}{f^{\prime}(s)}\right\}\left(y_{0}\right) \\
& =\frac{\left\{(t-s) f^{\prime}(t) f^{\prime}(s)+f(s) f^{\prime}(t)-f^{\prime}(s) f(t)\right\}^{2}}{f^{\prime}(s) f^{\prime}(t)\left(f^{\prime}(t)-f^{\prime}(s)\right)} .
\end{aligned}
$$

Second, we prove:

Lemma 6. The function $f$ satisfies the following:

$$
f(t) f^{\prime}(t)^{2}=4 a\left(t f^{\prime}(t)-f(t)\right)^{2},
$$

where $a$ is given by $f^{\prime \prime}(0)=2 a$.

Proof. Since the curve $X$ satisfies (1.3) with $\lambda=1 / 2$, we get $2 U(s, t)=T(s, t)$. By letting $s \rightarrow 0$, from (3.2) we get

$$
\epsilon \lim _{s \rightarrow 0} \frac{T(s, t)}{s}=\frac{f(t)}{2}
$$

where we use $f^{\prime}(0)=0$. From (3.4) we also get

$$
\begin{aligned}
2 \epsilon \lim _{s \rightarrow 0} \frac{U(s, t)}{s} & =\frac{\left\{f^{\prime \prime}(0) t f^{\prime}(t)-f^{\prime \prime}(0) f(t)\right\}^{2}}{f^{\prime \prime}(0) f^{\prime}(t)^{2}} \\
& =2 a \frac{\left\{t f^{\prime}(t)-f(t)\right\}^{2}}{f^{\prime}(t)^{2}}
\end{aligned}
$$

where we use $f^{\prime}(0)=0$ and $f^{\prime \prime}(0)=2 a>0$. Together with (3.6), (3.7) completes the proof.

Third, we prove: 
Lemma 7. The function $f$ satisfies the following:

$$
2 f(t)^{2} f^{\prime \prime}(t)=f^{\prime}(t)^{2}\left\{t f^{\prime}(t)-f(t)\right\} .
$$

Proof. By letting $s \rightarrow t$, we get from (3.2)

$$
\begin{aligned}
\epsilon \lim _{s \rightarrow t} \frac{T(s, t)}{s-t} & =\frac{1}{2} \lim _{s \rightarrow t} \frac{s f(t)-t f(s)}{s-t} \\
& =\frac{1}{2}\left(f(t)-t f^{\prime}(t)\right) .
\end{aligned}
$$

On the other hand, from (3.4) we get

$$
\begin{aligned}
2 \epsilon \lim _{s \rightarrow t} \frac{U(s, t)}{s-t} & =\lim _{s \rightarrow t} \frac{\left\{(t-s) f^{\prime}(t) f^{\prime}(s)+f(s) f^{\prime}(t)-f^{\prime}(s) f(t)\right\}^{2}}{(s-t) f^{\prime}(s) f^{\prime}(t)\left(f^{\prime}(t)-f^{\prime}(s)\right)} \\
& =-\frac{f(t)^{2} f^{\prime \prime}(t)}{f^{\prime}(t)^{2}} .
\end{aligned}
$$

Since $T=2 U$, together with (3.9), (3.10) completes the proof.

By eliminating $t f^{\prime}(t)-f(t)$ from (3.5) and (3.8), we get

$$
f^{\prime \prime}(t)=\frac{1}{4 \sqrt{a}} \frac{f^{\prime}(t)^{3}}{f(t)^{3 / 2}} .
$$

Letting $y=f(t)$, a standard method of ordinary differential equations using the substitution $w=d y / d t$ and $y^{\prime \prime}(t)=w(d w / d y)$ leads to

$$
d t=\left(\frac{1}{2 \sqrt{a y}}+c\right) d y
$$

where $c$ is a constant. Since $f(0)=0$, we obtain from (3.12)

$$
t=\frac{1}{\sqrt{a}}(\sqrt{y}+c y) .
$$

After replacing $t$ by $x$, we have for $y=f(x)$

$$
y= \begin{cases}\frac{2 \sqrt{a} c x+1-\sqrt{4 \sqrt{a} c x+1}}{2 c^{2}}, & \text { if } c \neq 0, \\ a x^{2}, & \text { if } c=0 .\end{cases}
$$

Note that

$$
f(0)=f^{\prime}(0)=0, f^{\prime \prime}(0)=2 a \quad \text { and } \quad f^{\prime \prime \prime}(0)=-12 \sqrt{a} a c \quad \text { or } \quad 0 .
$$

It follows from (3.14) that the curve $X$ around an arbitrary point $A$ is an open part of the parabola defined by

$$
a x^{2}-2 \sqrt{a} c x y+c^{2} y^{2}-y=0 .
$$

Finally using (3.15), in the same manner as in [6], we can show that the curve $X$ is globally an open part of a parabola. This completes the proof of Theorem 2. 


\section{Corollaries and examples}

In this section, we give some corollaries and examples.

Suppose that $X=X(s)$ is a strictly convex $C^{(3)}$ curve in the plane $\mathbb{R}^{2}$ which satisfies for all $s$ and sufficiently small $h_{i}, i=1,2$,

$$
U\left(s, h_{1}, h_{2}\right)=\lambda(s) T\left(s, h_{1}, h_{2}\right)^{\mu(s)},
$$

where $\lambda(s)$ and $\mu(s)$ are some functions. Then, in particular, for all $P=X(s)$ and sufficiently small $h>0$ the curve $X$ satisfies

$$
U_{P}(h)=\lambda(P) T_{P}(h)^{\mu(P)} .
$$

Using Theorem 1, by letting $h \rightarrow 0$ we see that $\mu(P)=1$. Hence, Theorem 1 again implies that $\lambda(P)=\frac{1}{2}$.

Thus, from Theorem 2 we get:

Corollary 8. Let $X$ denote a strictly convex curve in the plane $\mathbb{R}^{2}$. Then, the following are equivalent.

1) $X$ satisfies (4.1) for some functions $\lambda(s)$ and $\mu(s)$.

2) $X$ satisfies (4.1) with $\lambda=\frac{1}{2}$ and $\mu=1$.

3) $X$ is an open part of a parabola.

Finally, we give an example of a convex curve which satisfies

$$
U_{P}(h)=\frac{1}{2} T_{P}(h)
$$

for sufficiently small $h>0$ at every point $P \in X$, but it is not a parabola. Note that the example is not of class $C^{(2)}$, and hence it is not strictly convex either.

Example 9. Consider the graph $X$ of a function $f: \mathbb{R} \rightarrow \mathbb{R}$ which is given by for some positive distinct constants $a$ and $b$

$$
f(x)= \begin{cases}a x^{2}, & \text { if } x<0, \\ b x^{2}, & \text { if } x \geq 0 .\end{cases}
$$

It is straightforward to show that if $P$ is the origin, then for all $h$ we have

$$
U_{P}(h)=\frac{1}{2} T_{P}(h) .
$$

Hence $X$ satisfies $U_{P}(h)=T_{P}(h) / 2$ at the origin for all $h>0$. If $P \in X$ is not the origin, then there exists a positive number $\varepsilon(P)$ such that for every positive number $h$ with $h<\varepsilon(P), X$ satisfies $U_{P}(h)=T_{P}(h) / 2$.

Thus, $X$ satisfies $U_{P}(h)=T_{P}(h) / 2$ for sufficiently small $h>0$ at every point $P \in X$. But it is not a parabola. 


\section{References}

[1] W. A. Day, Inequalities for areas associated with conics, Amer. Math. Monthly 98 (1991), no. 1, 36-39.

[2] M. P. do Carmo, Differential Geometry of Curves and Surfaces, Prentice-Hall, Englewood Cliffs, NJ, 1976.

[3] D.-S. Kim and S. H. Kang, A characterization of conic sections, Honam Math. J. 33 (2011), no. 3, 335-340.

[4] D.-S. Kim and Y. H. Kim, Some characterizations of spheres and elliptic paraboloids, Linear Algebra Appl. 437 (2012), no. 1, 113-120.

[5] - Some characterizations of spheres and elliptic paraboloids II, Linear Algebra Appl. 438 (2013), no. 3, 1356-1364.

[6] - On the Archimedean characterization of parabolas, Bull. Korean Math. Soc. 50 (2013), no. 6, 2103-2114.

[7] D.-S. Kim, Y. H. Kim, and J. H. Park, Some characterizations of parabolas, Kyungpook Math. J. 53 (2013), no. 1, 99-104.

[8] J. Krawczyk, On areas associated with a curve, Zesz. Nauk. Uniw. Opol. Mat. 29 (1995), 97-101.

[9] B. Richmond and T. Richmond, How to recognize a parabola, Amer. Math. Monthly 116 (2009), no. 10, 910-922.

[10] S. Stein, Archimedes. What did he do besides cry Eureka?, Mathematical Association of America, Washington, DC, 1999.

DONG-SOO KIM

Department of Mathematics

Chonnam National University

KWANGJU 500-757, KOREA

E-mail address: dosokim@chonnam.ac.kr

KYU-CHul SHIM

Department of Mathematics

Chonnam National University

KWANGJU 500-757, KoreA

E-mail address: mathtsim@naver.com 